\title{
The role of the gamma function shape parameter in determining differences between condensation rates in bin and bulk microphysics schemes
}

\author{
Adele L. Igel and Susan C. van den Heever \\ Department of Atmospheric Science, Colorado State University, Fort Collins, Colorado, USA \\ Correspondence to: Adele L. Igel (aigel@ucdavis.edu)
}

Received: 22 January 2016 - Discussion started: 16 February 2016

Revised: 24 February 2017 - Accepted: 19 March 2017 - Published: 7 April 2017

\begin{abstract}
The condensation and evaporation rates predicted by bin and bulk microphysics schemes in the same model framework are compared in a statistical way using simulations of non-precipitating shallow cumulus clouds. Despite other fundamental disparities between the bin and bulk condensation parameterizations, the differences in condensation rates are predominantly explained by accounting for the width of the cloud droplet size distributions simulated by the bin scheme. While the bin scheme does not always predict a cloud droplet size distribution that is well represented by a gamma distribution function (which is assumed by bulk schemes), this fact appears to be of secondary importance for explaining why the two schemes predict different condensation and evaporation rates. The width of the cloud droplet size is not well constrained by observations, and thus it is difficult to know how to appropriately specify it in bulk microphysics schemes. However, this study shows that enhancing our observations of this width and its behavior in clouds is important for accurately predicting condensation and evaporation rates.
\end{abstract}

\section{Introduction}

Bin and bulk microphysics schemes are both popular approaches for parameterizing subgrid-scale cloud processes as evidenced by the large number of schemes that have been developed. Tables 2 and 3 in Khain et al. (2015) summarize the characteristics of dozens of microphysics schemes and discuss in detail the basic principles of the two basic types of schemes. Briefly, in double-moment bulk schemes, the mass mixing ratio and total number mixing ratio for predefined hy- drometeor species are predicted, and a function is assumed to describe the shape of the size distribution of each species. In contrast, bin schemes do not assume a size distribution function, but instead, the distribution is broken into discrete size bins, and the mass mixing ratio is predicted for each bin. Usually the size of each bin is fixed, in which case the number concentration is also known for each bin.

Bin schemes, particularly those for the liquid phase, are generally thought to describe cloud processes more realistically and accurately than bulk schemes, and thus they are often used as the benchmark simulation when comparing simulations with different microphysics schemes (e.g., Beheng, 1994; Seifert and Beheng, 2001; Morrison and Grabowski, 2007; Milbrandt and Yau, 2005; Milbrandt and McTaggartCowan, 2010; Kumjian et al., 2012). Bin schemes are much more computationally expensive since many additional variables need to be predicted. As a result, bin schemes are used less frequently. It is of interest, then, to see how well bulk and the more accurate liquid-phase bin microphysics schemes compare in terms of predicted process rates and to assess how much predictive value is added by using a bin instead of a bulk microphysics scheme. Furthermore, comparison of process rates in bin and bulk schemes could help to identify ways in which to improve bulk schemes.

One of the primary drawbacks of double-moment bulk schemes that assume probability distribution functions (PDFs) is that many microphysical processes are dependent on the distribution parameters that must be either fixed or diagnosed. In the case of a gamma PDF which is typically used in bulk schemes, this parameter is the shape parameter. The 


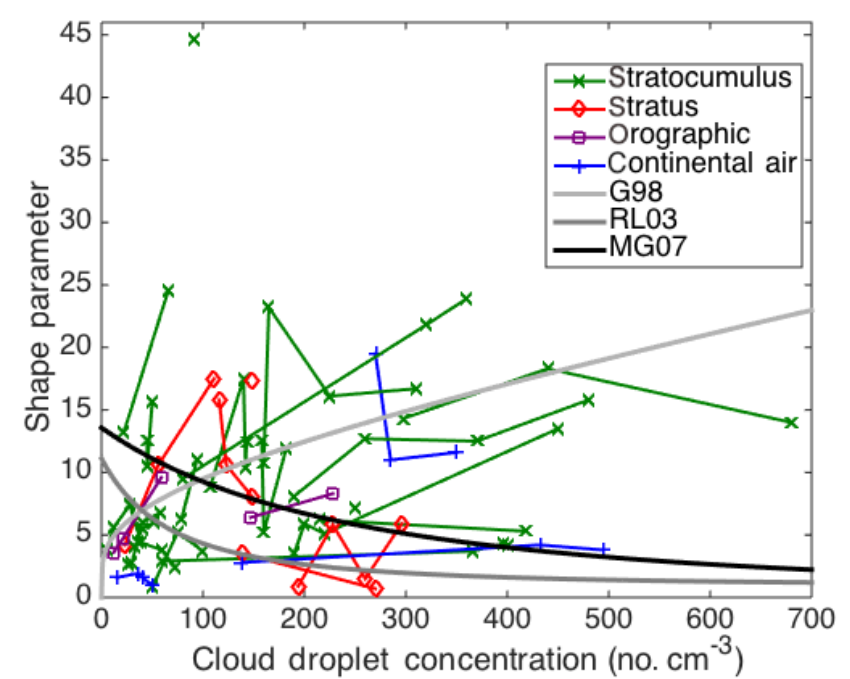

Figure 1. Shape parameter $(v)$ values as a function of cloud droplet concentration as reported by Miles et al. (2000) using 16 previous studies. Values, cloud classification, and groupings are based on their Tables 1 and 2. The solid gray lines and the black line show proposed relationships between the cloud droplet concentration and the shape parameter. G98 is from Eq. (9) in Grabowski (1998). RL03 is from Eq. (3) in Rotstayn and Liu (2003) with their $\alpha=$ 0.003. MG07 is from Eq. (2) in Morrison and Grabowski (2007). All equations were originally written for relative dispersion, which is equal to $v^{-1 / 2}$, and have been converted to equations for $v$ for this figure.

gamma size distribution $(n)$ is expressed as

$n(D)=\frac{N_{\mathrm{t}}}{D_{n}^{\nu} \Gamma(\nu)} D^{\nu-1} e^{-D / D_{n}}$,

where $v$ is the shape parameter, $N_{\mathrm{t}}$ is the total number mixing ratio, $D$ is the diameter, and $D_{n}$ is called the characteristic diameter. Much is still to be learned regarding what the most appropriate value of the shape parameter is and how it might depend on cloud microphysical properties.

Figure 1 shows previously proposed relationships between the cloud droplet number concentration and the shape parameter (Grabowski, 1998; Rotstayn and Liu, 2003; Morrison and Grabowski, 2007; hereinafter G98, RL03, and MG07, respectively) along with values of the shape parameter reported in the literature and summarized by Miles et al. (2000) for several different cloud types. The figure shows a wide range of possible values of the shape parameter based on observations. The lowest reported value is 0.7 and the highest is 44.6, though this highest point is clearly an outlier. Furthermore, there is no apparent relationship with the cloud droplet concentration in the data set as a whole, and both increases and decreases in the shape parameter are found with increasing droplet concentration among individual groupings. There is also no clear dependence of the shape parameter on cloud type. Figure 1 additionally shows that two of the proposed functions relating these two quantities are similar (RL03 and
MG07) but that the third function (G98) exhibits an opposite trend compared with these first two.

Furthermore, using appropriate values of the shape parameter may be necessary to accurately model cloud characteristics and responses to increased aerosol concentrations. Morrison and Grabowski (2007) found that switching from the MG07 to the G98 $N-v$ relationships in Fig. 1 led to a $25 \%$ increase in cloud water path in polluted stratocumulus clouds. This example shows that inappropriately specifying the shape parameter could have implications for the accurate simulation of not only basic cloud and radiation properties but also for the proper understanding of cloud-aerosol interactions. However, it is apparent from Fig. 1 that large uncertainties still exist regarding the behavior of the shape parameter and how it should be represented in models.

The goal of this study is to compare the condensation and evaporation rates predicted by bin and bulk microphysics schemes in cloud-resolving simulations run using the same dynamical and modeling framework and to assess what the biggest sources of discrepancies are. The focus is on condensation and evaporation since these processes occur in all clouds and are fundamental for all hydrometeor species. It will be shown that in spite of other basic differences between the particular bulk and bin microphysics schemes examined here, the lack of a prognosed shape parameter for the cloud droplet size distribution in the bulk scheme is often the primary source of differences between the two schemes. Thus, an improved understanding of the shape parameter is necessary from observations and models.

\section{Condensation and evaporation rate formulations}

The Regional Atmospheric Modeling System (RAMS) is used in this study. It contains a double-moment bulk microphysics scheme (BULK) (Saleeby et al., 2004; Saleeby and van den Heever, 2013) and the Hebrew University spectral bin model (BIN) (Khain et al., 2004). The Hebrew University spectral bin model is newly implemented in RAMS. Details about the implementation can be found in Appendix A.

In the BULK microphysics scheme, cloud droplet size distributions are assumed to conform to a gamma PDF given by Eq. (1). The condensation and evaporation scheme is described in detail in Walko et al. (2000), and the amount of liquid water condensed in a time step is given by their Eq. (6). Here, only the important relationships to the cloud droplet distribution properties are shown. Specifically, the BULK condensation and evaporation rate $\left(\partial r_{\mathrm{c}} / \partial t\right.$; time rate of change of the mass mixing ratio of cloud droplets) is proportional to $N_{\mathrm{t}}, \bar{D}$ (mass mean diameter), $v$, and $S$ in the following way:

$\frac{\partial r_{\mathrm{c}}}{\partial t} \propto(S-1) N_{\mathrm{t}} \bar{D} v\left(\frac{\Gamma(v)}{\Gamma(v+3)}\right)^{1 / 3}$ 
The BULK scheme does not use a saturation adjustment scheme for cloud water like many other bulk microphysics schemes do. Also, while not obvious here, the BULK scheme condensation and evaporation is implemented in such a way that evaporation cannot result in supersaturation, and likewise condensation cannot deplete the water vapor so much that the air is subsaturated at the end of the time step.

In contrast, the equation for the condensation and evaporation rate in the BIN is proportional to $S$ and the number concentration $N$ and diameter $D$ in each bin in the following way:

$\frac{\partial r_{\mathrm{c}}}{\partial t} \propto(S-1) \sum N_{i} D_{i}$.

As we would expect in a bin scheme, the condensation rate is proportional to the droplet properties in each bin rather than to the average droplet diameter and total number concentration. In the bin scheme, many small sub-time steps are taken during condensation and evaporation and the values of $S, N_{i}$, and $D_{i}$ are updated after each.

\section{Simulations}

In order to investigate the difference in condensation rates predicted by the two microphysics schemes, simulations of non-precipitating shallow cumulus clouds over land were performed. This cloud type was chosen in order to minimize the indirect impacts of precipitation processes. Furthermore, the daytime heating and evolution of the boundary layer results in a wider range of thermodynamic conditions than would occur in simulations of maritime clouds. The simulations were the same as those described in Igel and van den Heever (2017a, b). They were run with RAMS and employed $50 \mathrm{~m}$ horizontal spacing and $25 \mathrm{~m}$ vertical spacing over a grid that is $12.8 \times 12.8 \times 3.5 \mathrm{~km}$ in size. Such fine spacing was used in order to well resolve the cumulus clouds and their microphysical structure. The simulations were run for $9.5 \mathrm{~h}$ using a $1 \mathrm{~s}$ time step. Clouds appeared after about $4.5 \mathrm{~h}$. The simplified profiles of potential temperature, horizontal wind speed, and water vapor mixing ratio based on an Atmospheric Radiation Measurement (ARM) southern Great Plains (SGP) sounding from 6 July 1997 at 11:30 UTC (06:30 LST) presented in Zhu and Albrecht (2003) (see their Fig. 3) were used to initialize the model homogeneously in the horizontal direction. Random temperature and moisture perturbations were applied to the lowest model level at the initial time.

Some modifications were made to the model for this study only in order to make the two microphysics schemes more directly comparable. The diagnosis of saturation ratio from current values of the water vapor mixing ratio and temperature at the beginning of the microphysics routines was changed in the BULK scheme to make it the same as the calculation in the BIN. The BIN does not include a parameterization for aerosol dry deposition, so this process was turned off in the BULK scheme. Finally, the regeneration of aerosol upon droplet evaporation was deactivated in both microphysics schemes. Aerosol concentrations were initialized homogeneously in the horizontal and vertical directions. Aerosol particles did not interact with radiation.

Five simulations were run with the BULK scheme and three with the BIN scheme. Since the relationships in Fig. 1 (G98; RL03; MG07) suggest that the shape parameter may depend on the cloud droplet number concentration, the simulations were run with three different aerosol concentrations, specifically, 100, 400, and $1600 \mathrm{~cm}^{-3}$, in order to obtain a larger range of droplet concentration values. The aerosol in the BIN simulations was initialized with, and in the BULK simulations was assumed to follow, a lognormal distribution with a median radius of $40 \mathrm{~nm}$ and a spectral width of 1.8 . These BULK simulations used a shape parameter value of 4 . Two additional BULK simulations were run with an aerosol concentration of $400 \mathrm{~cm}^{-3}$ and shape parameter values of 2 and 7. These values were chosen based on previous analysis of the BIN simulations in Igel and van den Heever (2017a). The BIN simulations will be referred to by the microphysics scheme abbreviation and the initial aerosol concentration, e.g., BIN100, and the BULK simulation names will additionally include the value of the cloud droplet shape parameter, e.g., BULK100-NU4.

\section{Results}

\subsection{Instantaneous condensation rates}

In order to compare directly the condensation rates predicted by the BULK and BIN microphysics schemes, it is necessary to evaluate these rates given the same thermodynamic and cloud microphysical conditions. The BULK condensation equation (Eq. 2) is approximately linearly proportional to four quantities: $S, N_{\mathrm{t}}, \bar{D}$, and $v$. We say approximately proportional since the presence of the ventilation coefficient (which itself depends on $\bar{D}$ and $v$ ) makes these factors not truly proportional to the condensation rate. In the BIN scheme, among these four variables, the condensation rate is only explicitly proportional to $S$ and is not explicitly proportional to $N_{\mathrm{t}}, \bar{D}$, or $v$ (which do not appear at all in Eq. 3) since the BIN scheme does not make assumptions about the functional form of the size distribution. If it is assumed nevertheless that the BIN size distributions can be described by some probability distribution function (which does not necessarily have to be a gamma distribution), then we would still expect the BIN scheme condensation rate to scale linearly with $N_{\mathrm{t}}$ and $\bar{D}$.

Therefore, in order to best compare the condensation rates between the two schemes, the condensation and evaporation rates that occur during one time step were binned by the values of $S, N_{\mathrm{t}}$, and $\bar{D}$ that existed at the start of the condensation and evaporation process and were averaged in each bin. 

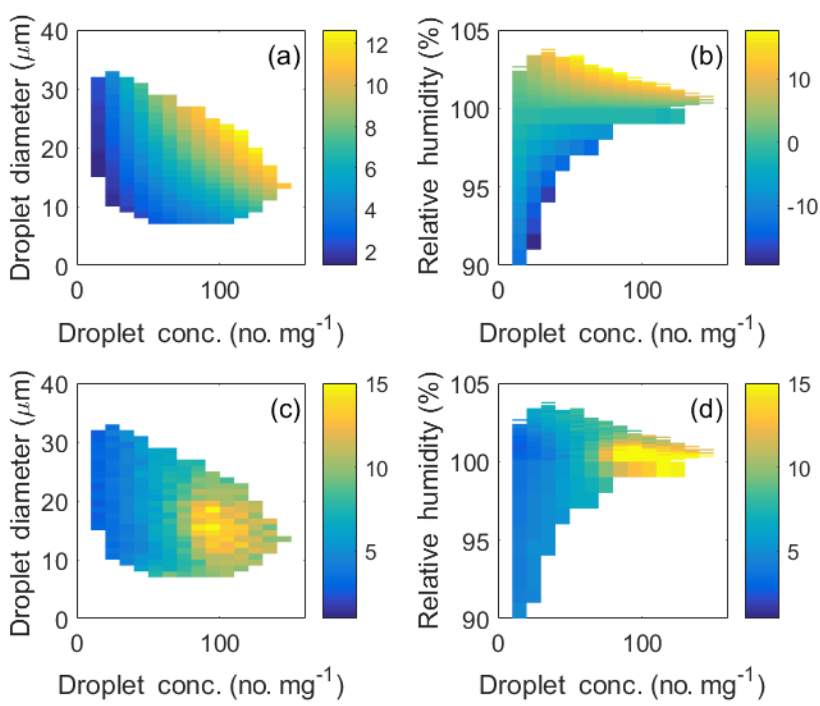

Droplet conc. (no. $\mathrm{mg}^{-1}$ )

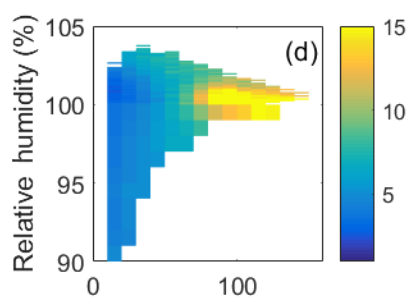

Droplet conc. (no. $\mathrm{mg}^{-1}$ )

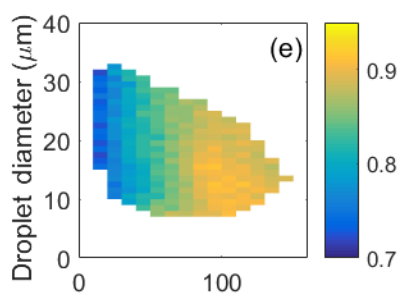

Droplet conc. (no. $\mathrm{mg}^{-1}$ )

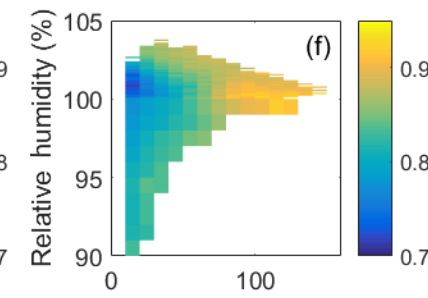

Droplet conc. (no. $\mathrm{mg}^{-1}$ )

Figure 2. (a, b) Example average condensation and evaporation rates $\left(\mathrm{mg} \mathrm{kg}^{-1} \mathrm{~s}^{-1}\right),(\mathbf{c}, \mathbf{d})$ example average shape parameters, and $(\mathbf{e}, \mathbf{f})$ example average values of $\overline{f_{\mathrm{NU}}, \mathrm{BIN}}$ in joint bins from BIN400. Panels $(\mathbf{a}, \mathbf{c}, \mathbf{e})$ show average values of the two quantities for all joint bins from BIN400 with $S$ between 1.011-1.012, and (b, d, f) show averages for all joint bins from BIN400 with $\bar{D}$ between 19 and $20 \mu \mathrm{m}$.

(Note that these phase space bins are not the same as the hydrometeor distribution bins.) That is, all points with the same $S, N_{\mathrm{t}}$, and $\bar{D}$ were grouped and the average condensation or evaporation in each group of points was calculated. The average condensation rate in each $S, N_{\mathrm{t}}$, and $\bar{D}$ joint bin was calculated separately for each simulations.

Examples of the average condensation and evaporation rates from BIN400 are shown in Fig. 2a-b as functions of $S, N_{\mathrm{t}}$, and $\bar{D}$. Values in each joint bin differ for the other simulations. Saturation ratio bin widths of 0.1 or 1 were used where the cloud was supersaturated or subsaturated, respectively. For $\bar{D}$, bin widths of $1 \mu \mathrm{m}$ were used. For $N$, the bin width depended on the initial aerosol concentration of the simulation: bin widths of $2.5,10$, and $40 \mathrm{mg}^{-1}$ were used for simulations with an initial aerosol concentration of 100 , 400 , and $1600 \mathrm{mg}^{-1}$, respectively. The output from the dynamical model only includes the values of $S, N_{\mathrm{t}}$, and $\bar{D}$ after condensation and evaporation have occurred. However, since the rates of condensation and droplet nucleation were known from additional model output and since microphysics
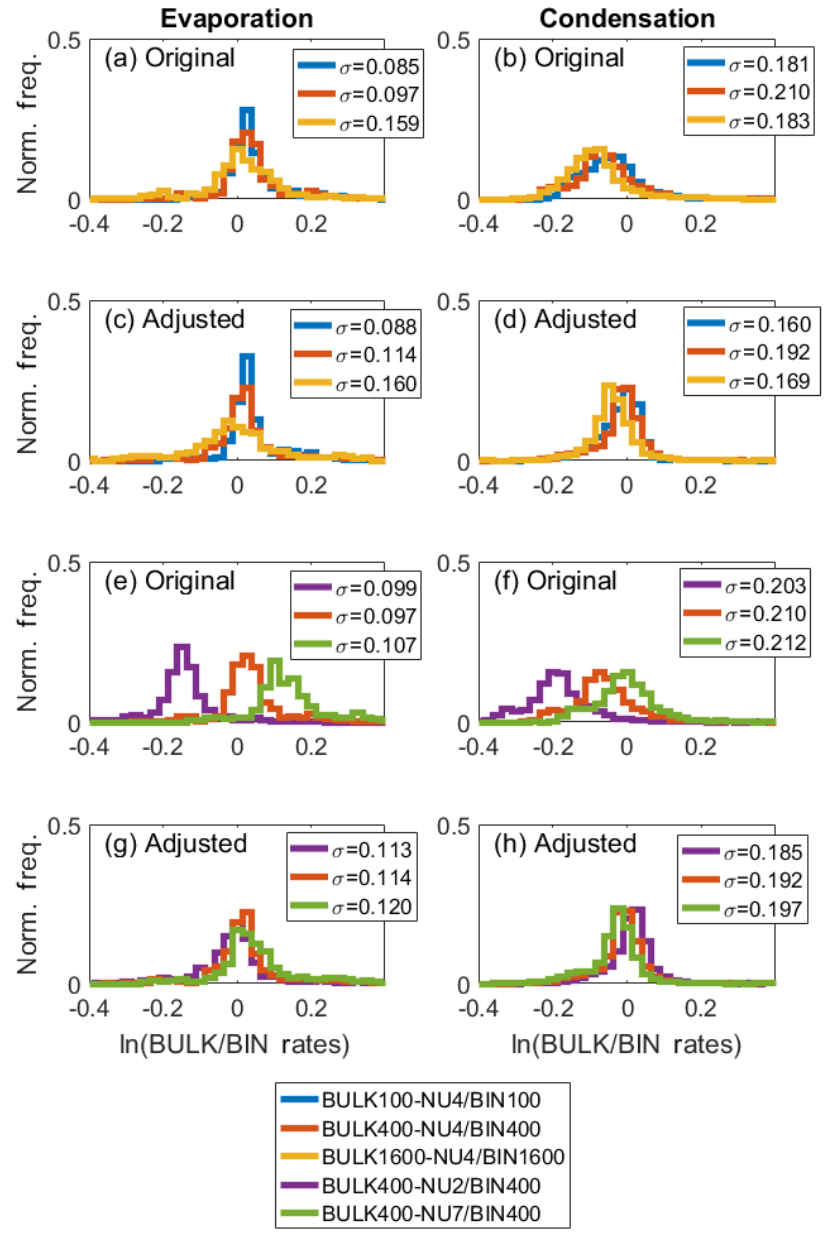

Figure 3. The ratio of the BULK to BIN (a-c) condensation and (d-f) evaporation rates as a function of saturation ratio $(S)$ and integrated diameter $(N \bar{D})$ for each pair of simulations. Note the differences in axes limits.

was the last physical process to occur during a time step in RAMS, the $S, N_{\mathrm{t}}$, and $\bar{D}$ that existed before condensation occurred were easily calculated from the model output. All points where the cloud mixing ratio before condensation was greater than $0.01 \mathrm{~g} \mathrm{~kg}^{-1}$ and the cloud droplet number concentration was greater than $5 \mathrm{mg}^{-1}$ were included in the analysis. Finally, joint bins with fewer than 50 data points were discarded.

As seen in Fig. 2a-b, there is a smooth transition to higher condensation rates as the saturation ratio increases and to higher condensation $(S \geq 1)$ and evaporation $(S<1)$ rates as the diameter or number mixing ratio increases. This is expected based on the condensation equations (Eqs. 2, 3). All other simulations behave similarly.

Note that the aerosol activation parameterizations in the BULK and BIN microphysics were not the same, and hence the number of nucleated cloud droplets was not the same. This impacted the number of data points within each joint 
$S, N_{\mathrm{t}}$, and $\bar{D}$ bin. However, we are primarily concerned with the average condensation rate in each joint bin, and the average value should not be impacted by the number of data points within a bin provided that the number is sufficiently high (joint bins with fewer than 50 data points are neglected). Therefore, the differences in the aerosol activation parameterizations, or for that matter, differences in the evolution of the cloud fields, should not influence the average condensation rates as evaluated in our framework.

In order to compare easily the condensation rates predicted by the two microphysics schemes, we calculate the ratio of the average condensation or evaporation rate of each joint bin from a BULK simulation to the average condensation or evaporation rate of the corresponding joint bin from a BIN simulation and then calculate the natural logarithm of each ratio. These will be referred to as "In(ratios)". We find the $\ln$ (ratios) of the average condensation and evaporation rates for five pairs of simulations. Specifically, BULK400-NU2, BULK400-NU4, and BULK400-NU7 are all compared to BIN400, while BULK100-NU2 is compared to BIN100 and BULK1600-NU2 is compared to BIN1600. Histograms of the $\ln$ (ratios) for all pairs of simulations are shown in Fig. 3a, $\mathrm{b}$, e, and $\mathrm{f}$. The data have been separated into subsaturated (evaporating) and supersaturated (condensing) points. Positive values indicate that the rates in the BULK scheme are larger, and negative values indicate that the rates in the BIN scheme are larger. Values of $\pm 0.1( \pm 0.2)$ correspond to about a $10 \%(20 \%)$ difference in the condensation or evaporation rate between the two schemes for the joint bin.

First we examine the impacts of increasing aerosol concentrations on the agreement of evaporation and condensation rates in BULK and BIN simulations. Figures 3ab show the histograms of the condensation and evaporation rate $\ln$ (ratios) for BULK100-NU4 compared to BIN100, BULK400-NU4 compared to BIN400, and BULK1600-NU4 compared to BIN1600. Figure $3 \mathrm{~b}$ reveals that in general the condensation rate is higher in the BIN scheme simulations as indicated by the more frequent negative $\ln$ (ratios). On the other hand, the evaporation rates are more similar between the two schemes as indicated by the most frequent $\ln$ (ratios) being equal to or slightly greater than 0 in Fig. 3 a.

Figure $3 \mathrm{e}-\mathrm{f}$ show the histograms of condensation and evaporation rate $\ln$ (ratios) for the three BULK400 simulations with different values of the shape parameter, all compared to BIN400. For both condensation and evaporation, the peak of the $\ln$ (ratios) histograms increases as the cloud droplet shape parameter used in the BULK400 simulations increases. For the BULK400-NU2 simulation, the condensation and evaporation rates are frequently $20 \%$ lower than the BIN400 rates or more, whereas for the BULK400-NU7 simulation, the condensation rates compared to the BIN400 simulation are most frequently very similar ( $\ln ($ ratios) near zero). Thus, the value of the cloud droplet shape parameter chosen for use in a simulation is clearly important for determining how well a bulk microphysics scheme compares to a bin microphysics scheme in terms of predicted condensation and evaporation rates.

\subsection{Accounting for the shape parameter}

Fortunately, we know theoretically how the cloud droplet shape parameter will alter condensation and evaporation rates, and this dependency can be accounted for in our comparison of the two microphysics schemes. The shape parameter term in Eq. (2) (hereafter $f_{\mathrm{NU}}$ ), which is equal to $v\left(\frac{\Gamma(v)}{\Gamma(v+3)}\right)^{1 / 3}$, indicates that when a gamma PDF is assumed, the condensation rate is proportional to the shape parameter $v$ such that a higher shape parameter results in higher condensation rates. Of course, the BIN scheme makes no assumptions about the size distribution functionality and its condensation scheme does not depend on the shape parameter. However, in order to characterize the shape of the predicted BIN cloud droplet size distributions and to facilitate the comparison of the BIN and BULK condensation rates, we assumed that the predicted BIN size distributions are gamma PDF-like and found the best-fit gamma PDF parameters (see Eq. 1) for the cloud droplet size distributions at every cloudy grid point in the BIN simulations.

In order to find the best-fit shape parameters, we defined cloud droplets as belonging to one of the first 15 bins of the BIN liquid array (the remaining 18 bins contain raindrops), which corresponded to a maximum cloud droplet diameter of $50.8 \mu \mathrm{m}$. Many methods are available to find such best-fit parameters, but they generally all give similar results (McFarquhar et al., 2015). Here we used the maximumlikelihood estimation (MLE) method. For our problem, the $\log$-likelihood function $(\ln (L))$ is defined as

$\ln L=\frac{1}{N_{\mathrm{t}}} \sum_{i=1}^{15} N_{i} \ln n\left(D_{i}\right)$,

where $n\left(D_{i}\right)$ is the value of the gamma PDF (Eq. 1) for $D_{i}$ with unknown values of the parameters $D_{n}$ and $v$. The function is normalized by the total cloud droplet concentration $N_{\mathrm{t}}$ in order to remove $N_{\mathrm{t}}$ as a free parameter in Eq. (1). As indicated by its name, the MLE method seeks to maximize the log-likelihood function given by Eq. (4). To do so, we used the MATLAB function fmincon to find the parameter values that minimized $-1 \cdot L$.

Note that while we could determine the values of $S, N_{\mathrm{t}}$, and $\bar{D}$ that existed before condensation occurred, we could not determine the value of the best-fit shape parameter for this time because the change in mixing ratio of each bin was not output by RAMS. Thus, the average shape parameters used in the analysis are those that exist at the end of the time step. Nonetheless, given the short time step used in these simulations, it was not expected that the best-fit shape parameter would change much in one time step in most cases. The exception may be for very broad distributions characterized by low shape parameters. In part due to this concern, cloudy 
points with best-fit shape parameters less than 1 are not included in the analysis. This criterion eliminated 4.5, 5.1, and $8.6 \%$ of the data in BIN100, BIN400, and BIN1600, respectively. Overall, the impact of using the post-condensation shape parameters is not expected to have a large impact on the results. Examples of the average shape parameters in each joint bin are shown in Fig. 2c-d. The shape parameter tends to increase with droplet concentration and be low (5 or less) for relative humidity less than $99 \%$. In-depth analysis of the best-fit shape parameter in the BIN simulations is found in Igel and van den Heever (2017a).

Using these best-fit shape parameters from the BIN simulations and the specified shape parameters from the BULK simulations, the shape parameter term $\left(f_{\mathrm{NU}}\right)$ can be evaluated for each cloudy point for all simulations. In the case of each BULK simulation, the value of $f_{\mathrm{NU}, \mathrm{BULK}}$ is the same for every cloudy point since the value of $f_{\mathrm{NU}, \mathrm{BULK}}$ is uniquely determined by the choice of the shape parameter value. Specifically, $f_{\mathrm{NU}, \mathrm{BULK}}=0.69,0.81$, and 0.88 for NU2, NU4, and NU7 simulations, respectively. For the BIN simulations, $f_{\mathrm{NU}, \mathrm{BIN}}$ can be calculated using the best-fit shape parameters and will have a different value for every cloudy grid point. The values of $f_{\mathrm{NU}, \mathrm{BIN}}$ for the cloudy grid points in each joint bin were averaged together to find a mean $\overline{f_{\text {NU,BIN }}}$ for each joint $S, N_{\mathrm{t}}$, and $\bar{D}$ bin for each BIN simulation. Example values of $\overline{f_{\mathrm{NU}, \mathrm{BIN}}}$ for some joint bins are shown in Fig. 2e-f. We can use the values of $f_{\mathrm{NU}, \mathrm{BULK}}$ and $\overline{f_{\mathrm{NU}, \mathrm{BIN}}}$ to account for the differences in condensation and evaporation rates between the two schemes that arise due to different shape parameters. Specifically, in our analysis, we adjusted the mean condensation and evaporation rates $(C)$ for each joint bin from the BULK simulations in the following way:

$\overline{C_{\mathrm{BULK}, \text { adjusted }}}=\overline{C_{\mathrm{BULK}, \text { original }}} \frac{\overline{f_{\mathrm{NU}, \mathrm{BIN}}}}{f_{\mathrm{NU}, \mathrm{BULK}}}$.

Note again that the value of $\overline{f_{\mathrm{NU}, \mathrm{BIN}}}$ will be different for each joint bin. By making this correction, we found the condensation and evaporation rates that the BULK simulations would have had if they had used the same value of the shape parameter that best characterized the cloud droplet size distributions that were predicted by the BIN simulations. To be clear, we did not run new simulations; rather, the outputted condensation and evaporation rates from the existing BULK simulations were adjusted for the purposes of our analysis using Eq. (5) to account for the differences in size distribution shapes between the BIN and BULK simulations. We will next compare these adjusted BULK condensation and evaporation rates to the BIN rates to see if the comparison improves.

The $\ln$ (ratios) of the adjusted condensation and evaporation rates from the BULK simulations to the rates from the BIN simulations are shown in Fig. 3c-d and g-h. Hereafter, these $\ln$ (ratios) will be called adjusted $\ln$ (ratios). The most frequent value of the adjusted $\ln$ (ratios) is near zero (indicating that the two schemes predict the same rate) for all simulation pairs and for both condensation and evaporation. The impact of the adjustment is most notable in Fig. $3 \mathrm{~g}-\mathrm{h}$ where the histograms of the adjusted $\ln$ (ratios) now nearly lie on top of one another, whereas in Fig. 3e-f they are clearly separated. Thus, it appears that our method of accounting for the value of the shape parameter has worked well.

Additionally, the standard deviations of the adjusted $\ln$ (ratio) histograms (shown in the legend of each panel) for condensation are decreased slightly. This is not the case for the adjusted $\ln$ (ratio) histograms for evaporation, where for all simulation pairs the standard deviation is increased compared to the original $\ln$ (ratio) histograms. Nonetheless, given that all adjusted histograms (Fig. 3c-d, g-h) now have a modal value near 0 , whereas this was not the case with the original histograms (Fig. 3a-b, e-f), the shape parameter appears to be the primary reason why the condensation and evaporation rates in the two schemes do not always agree.

\subsection{Other considerations}

While the shape parameter appears to be the primary cause of differences in condensation and evaporation rates in bin and bulk microphysics schemes, it is worth investigating which other factors are important.

\subsubsection{Relative humidity}

When the relative humidity is close to $100 \%$, the condensation and evaporation rates are limited by the small supersaturation or subsaturation. In these situations, the droplet properties are expected to have little impact on the condensation or evaporation rate. Instead, these rates will be largely determined by how the schemes behave when the timescale for condensation or evaporation is smaller than the time step of the model. Figure 4 shows the average and standard deviation of the adjusted $\ln$ (ratios) for all five pairs of simulations as a function of relative humidity. Both the average and the standard deviation peak for relative humidity near $100 \%$. This indicates that the agreement between the bulk and bin schemes on condensation and evaporation rates is poor, just as we expected it to be based on the above arguments. That said, condensation and evaporation rates occurring with relative humidity near $100 \%$ are small in magnitude, and disagreements here are not expected to have a large impact on the simulation evolution.

We repeated the analysis shown in Fig. 3 but excluding data points where the relative humidity before condensation and evaporation was between 99.5 and $100.5 \%$. The results are shown in Fig. 5. Qualitatively, the results in Figs. 3 and 5 are similar. The adjusted histograms are all centered near 0 , but the decrease in the standard deviation of the $\ln$ (ratios) (shown in the legends) from Fig. 3 to 5 is substantial. This indicates that by removing cloudy points with relative hu- 

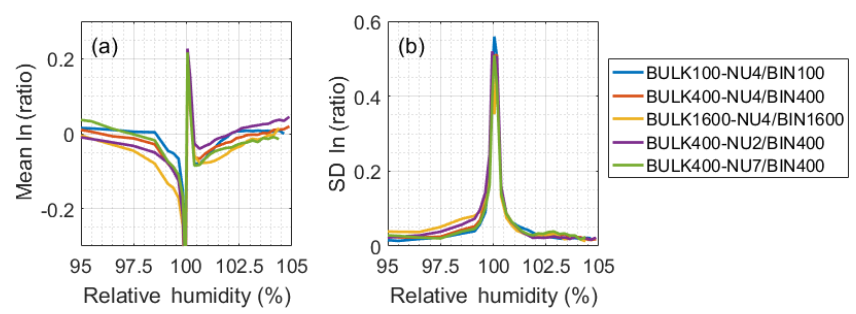

Figure 4. The (a) mean $\ln$ (ratio) and (b) standard deviation of the $\ln$ (ratios) as a function of relative humidity for all five simulation pairs.

midity between 99.5 and $100.5 \%$, the agreement between the two schemes increases. That said, the standard deviations of the adjusted evaporation histograms are still higher than those of the original histograms. Finally, unlike in Fig. 3, the standard deviation for the adjusted condensation histograms is consistently lower than that of the evaporation histograms. Thus, overall, it seems that the correction based on the shape parameter for condensation is more successful than that for evaporation in terms of the spread of $\ln$ (ratios). Potential reasons for this difference are explored next.

\subsubsection{Appropriateness of the gamma PDF and fractional mass change}

One potential reason worth considering is that the gamma PDF is not always appropriate for characterizing the cloud droplet size distributions in the BIN simulations. The BIN microphysics scheme is capable of predicting any shape for the cloud droplet size distributions, including size distributions that may be bimodal. To assess how well our fitted gamma PDFs approximated the actual simulated cloud droplet size distributions, we calculated the normalized root mean square error (NRMSE) of the fits using MATLAB's goodnessOfFit function. An NRMSE of 1 indicates that the fit was no better than a flat line equal to the mean of the size distribution, and a value of 0 indicates a perfect fit. Figures $6 a-b$ show cumulative histograms of the NRMSE values from the three BIN simulations for both evaporating and condensing cloudy points. Note that these are not cumulative histograms of mean values from joint bins as in Fig. 3, but rather they are cumulative histograms of the NRMSE values at all individual cloudy grid points in the BIN simulations. The majority of grid points have NRMSE values between about 0.4 and 0.6 , which indicates that in general the gamma PDF characterizes the simulated cloud droplet size distributions moderately well. The cumulative distributions of NRMSE are similar for all three BIN simulations and similar for evaporating and condensing cloudy grid points. This suggests that the NRMSE probably cannot explain why the correction in Fig. 5 leads to a reduction in the standard deviation of $\ln$ (ratios) for condensation but to an increase in the standard deviation of $\ln$ (ratios) for evaporation. Nonetheless,
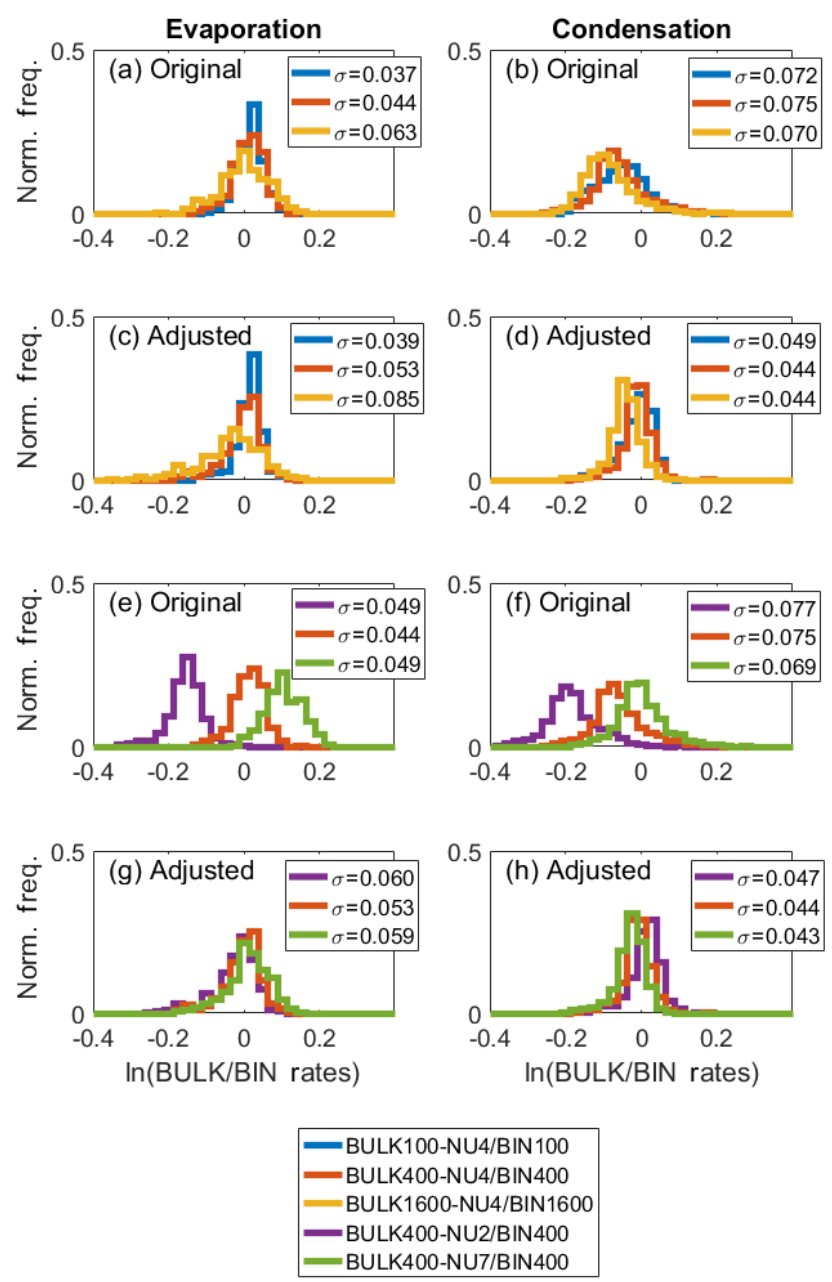

Figure 5. Like Fig. 3 but excluding grid points from the joint bins with relative humidity between 99.5 and $100.5 \%$.

we still expect that higher NRMSE should result in differences between the condensation and evaporation rates in bin and bulk schemes. This will be discussed further below.

Another potential reason that evaporation and condensation comparisons are different relates to the fractional change of mass. Specifically, the comparison may be better for situations in which only a small fraction of the total cloud droplet mass is evaporated or condensed within a time step versus a situation in which a large fraction of mass is evaporated or condensed. The reason the fractional change in mass may be important is related to the different treatments of the time step during condensation and evaporation in the two schemes. The BIN microphysics scheme takes an iterative approach to condensation and evaporation in which many small steps are taken. After each small step the droplet properties are updated. When the droplet properties are changing rapidly, this approach may be important for accurately predicting the evolution of the total mass and number of cloud droplets. On the other hand, the RAMS bulk scheme 

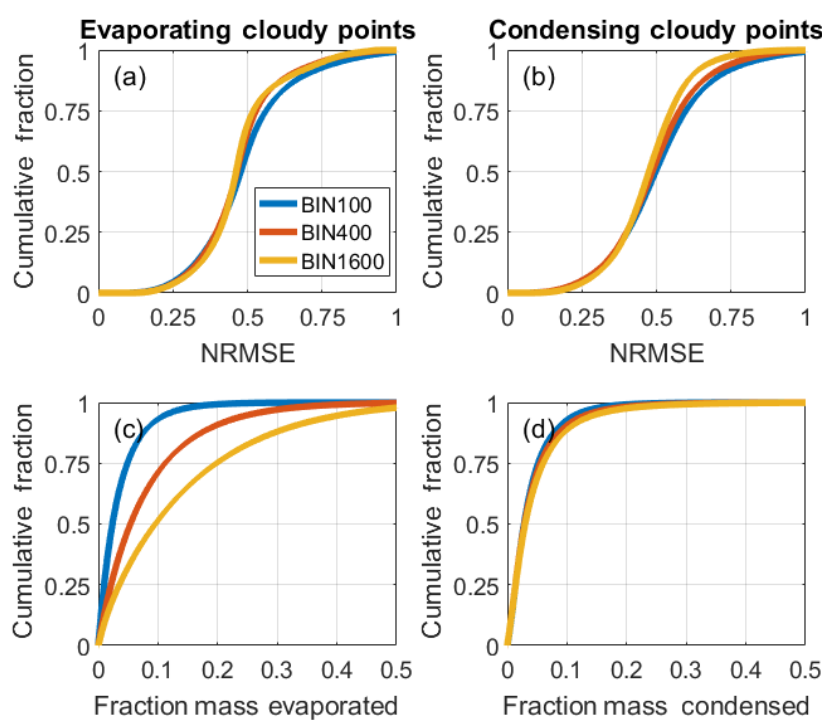

Figure 6. Cumulative distributions of (a, b) NRMSE, (c) fraction of mass evaporated, and (d) fraction of mass condensed. Panels (a, c) include only grid points where evaporation occurred and $(\mathbf{b}, \mathbf{d})$ include only grid points where condensation occurred.

takes just one step (which is equal to the full model time step length) and cannot account for rapidly changing droplet properties within the time step.

Cumulative histograms of the fraction of cloud mass evaporated in one full time step are shown in Fig. 6c for the BIN simulations. Higher fractions of mass are evaporated more frequently as the initial aerosol concentration increases. This result is not surprising given that the high numbers of cloud droplets nucleated from the high numbers of aerosol particles will induce on average higher evaporation rates (Eqs. 2 and 3) that cause a higher fraction of mass to be evaporated in one time step. Similarly, cumulative histograms of the fraction of cloud droplet mass condensed in the time step are shown in Fig. 6d. Again, high fractions of cloud mass are condensed more frequently as the initial aerosol concentration increases. In general, large fractional changes in the cloud mass are more frequent during evaporation than during condensation. This suggests that the fractional mass change may be a reason for the better comparison of condensation rates than evaporation rates in Fig. 5 after the shape parameter correction was applied.

To explore simultaneously the impact of NRMSE and fractional mass change on the comparison of bin and bulk scheme condensation and evaporation rates, we also calculated the mean NRMSE and fractional mass change of each of the joint $S, N_{\mathrm{t}}$, and $\bar{D}$ bins in addition to the adjusted $\ln$ (ratio) for each bin that we have shown previously. In this analysis, we have excluded points with relative humidity between 99.5 and $100.5 \%$ based on our previous analysis of the impact of relative humidity. Joint bins with similar mean NRMSE and fractional mass change were grouped together

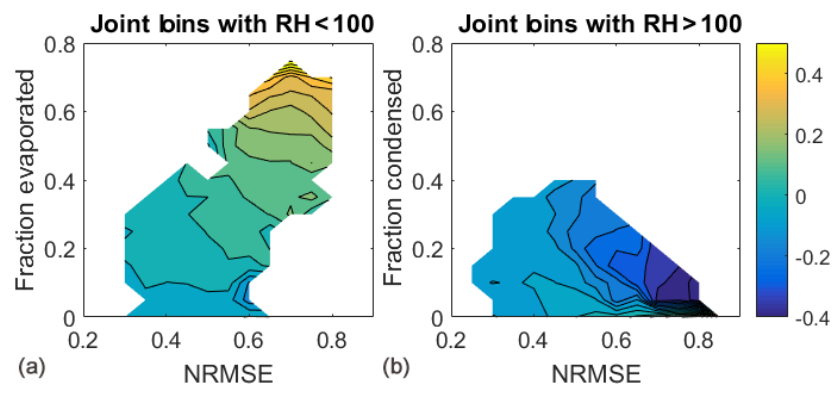

Figure 7. For each joint $S, N_{\mathrm{t}}$, and $\bar{D}$ bin, the mean NRMSE and mean fraction of mass evaporated or condensed was calculated. Each panel shows the relationship between the mean NRMSE, mean adjusted $\ln$ (ratio) (colors), and (a) mean fraction of mass evaporated or (b) mean fraction of mass condensed. Joint bins from all simulation pairs are included in the mean adjusted $\ln$ (ratios) that are shown.

and the mean adjusted $\ln$ (ratios) for each group was calculated. Joint bin pairs from all simulation pairs were included. The results are shown in Fig. 7, again for condensation and evaporation separately, where colors show the mean of the adjusted $\ln$ (ratios) as a function of NRMSE and fractional mass change. Colors near zero (teal) indicate that the two schemes agree well after the shape parameter correction is applied, whereas colors away from zero (blue and yellow) indicate that the two schemes do not agree well even after the shape parameter adjustment is applied.

Evaporation will be considered first (Fig. 7a). For evaporated mass fraction less than about 0.3 , the mean adjusted $\ln$ (ratios) are near zero. As the evaporated mass fraction increases above 0.3 , the NRMSE also begins to increase, which makes it difficult to understand the influence of either the NRMSE or evaporated mass fraction on the scheme comparison by looking at them in isolation. However, by looking at them together in Fig. 7a, we see that the evaporated mass fraction seems to be driving the increase in the adjusted mean $\ln$ (ratio) away from 0 , particularly when the evaporated mass fraction is greater than 0.4 . For these values, the contour lines are approximately flat, which indicates that there is little dependence of the mean adjusted $\ln$ (ratios) on NRMSE.

The NRMSE seems to be more important for condensation than evaporation. As the NRMSE increases above about 0.5 in Fig. $7 \mathrm{~b}$ for condensation, the mean adjusted $\ln$ (ratios) begin to drop away from zero, and the two schemes have worse agreement on the condensation rates. Like for evaporation, when NRMSE and the condensed mass fraction are both relatively low, the mean adjusted $\ln$ (ratios) are near zero and show little dependence on NRMSE or fractional mass change. 


\section{Conclusions}

In this study, we have compared the cloud condensation rates predicted by a bulk and a bin microphysics scheme in simulations of non-precipitating cumulus clouds run using the same dynamical framework, namely RAMS. The simulations were run with three different background aerosol concentrations in order to consider a large range of microphysical conditions. Two additional simulations with the RAMS bulk microphysics scheme were run with different settings for the cloud droplet shape parameter.

When the condensation and evaporation rates were binned by saturation ratio, cloud droplet number concentration, and mean diameter, the BULK rates were on average higher or lower depending primarily on the value of the shape parameter used in the BULK simulations. Since the theoretical relationship between the shape parameter and condensation and evaporation rates is known, we adjusted the BULK rates to be those that the simulations would have predicted if they had used the same value of the shape parameter as was found by fitting gamma PDFs to the BIN droplet size distribution output. After doing so, we showed that the BULK and BIN rates were in general in much better agreement, although the condensation rates agreed better than the evaporation rates.

Other factors were also suggested to impact the agreement of condensation and evaporation rates in the BIN and BULK simulations. First, the agreement was worse as the relative humidity approached $100 \%$. Second, when the simulated binned size distributions did not conform closely to a gamma PDF (NRMSE was high), the agreement was also worse, particularly for condensation. Lastly, when a large fraction of the cloud droplet mass was evaporated or condensed within a model time step, the agreement was also worse, particularly for evaporation. We hypothesize that the reason for a dependence on the fractional mass change is related to the different approaches taken by the BIN and BULK schemes to solve the condensation equation. However, all three of these factors were found to be of secondary importance compared to the shape parameter.

Again, it appears that when the relative humidity is not near $100 \%$, the most important factor for agreement in cloud droplet condensation and evaporation rates between bin and bulk schemes is the shape of the cloud droplet size distribution. More effort is needed to understand the cloud droplet shape parameter in order to improve the representation of cloud droplet size distributions in bulk microphysics schemes. Improvement in the representation of size distributions should lead to better agreement in the simulated macroscopic properties of clouds by the two schemes, although such potential for better agreement has not been shown here. Finally, while the methods we have used to here to demonstrate the importance of the shape parameter were effective, we are not suggesting that the same methods would be best for improving bulk schemes.
Although we have only investigated two specific schemes, it is expected that the results can be applied more generally to bin and bulk schemes that do not use saturation adjustment. Additional work should be conducted using a similar approach in order to compare and evaluate additional microphysics schemes and additional microphysical processes. While it is clear that the shape parameter explains much of the discrepancies in predicted condensation rates between bin and bulk schemes, our understanding of what the most appropriate value of the shape parameter is or how it should vary as a function of basic cloud properties is limited. More work then is also needed to understand cloud droplet distribution width from observations and measurements.

Data availability. The simulations are archived at Colorado State University and are available upon request. 
Appendix A: Implementation of the Hebrew University BIN scheme into RAMS

While the present study is only concerned with warm-phase processes, the methods to interface the Hebrew University BIN scheme with the RAMS radiation scheme (Harrington, 1997) will be described here. The RAMS radiation scheme uses precomputed lookup tables for the extinction coefficient, single-scattering albedo, and asymmetry parameter for each hydrometeor species. All liquid drops are represented as one species in the BIN, so these liquid bins are classified as either cloud droplets or raindrops using the same size threshold used by the RAMS microphysics scheme to distinguish between these two species. For each set of BIN bins that corresponds to a RAMS species, the total number concentration and mean diameter are calculated, a gamma distribution shape parameter of 2 is assumed, and the appropriate set of look-up tables for the corresponding RAMS species is used for all radiative calculations. 
Competing interests. The authors declare that they have no conflict of interest.

Acknowledgements. The authors thank Alexander Khain for generously sharing his BIN code in order to make this study possible. The authors also thank the reviewers for providing useful comments that led to improvements in this paper. This material is based on work supported by the National Science Foundation Graduate Research Fellowship Program under Grant No. DGE-1321845 and the National Aeronautics and Space Administration Grant No. NNX13AQ32G. Additional information can be requested from the corresponding author.

Edited by: E. Jensen

Reviewed by: three anonymous referees

\section{References}

Beheng, K. D.: A parameterization of warm cloud microphysical conversion processes, Atmos. Res., 33, 193-206, doi:10.1016/0169-8095(94)90020-5, 1994.

Grabowski, W. W.: Toward Cloud Resolving Modeling of Large-Scale Tropical Circulations: A Simple Cloud Microphysics Parameterization, J. Atmos. Sci., 55, 3283-3298, doi:10.1175/1520-0469(1998)055<3283:TCRMOL>2.0.CO;2, 1998.

Harrington, J. Y.: The effects of radiative and microphysical processes on simulation of warm and transition season Arctic stratus, Colorado State University, 1997.

Igel, A. L. and van den Heever, S. C.: The Importance of the Shape of Cloud Droplet Size Distributions in Shallow Cumulus Clouds. Part I: Bin Microphysics Simulations, J. Atmos. Sci. 74, 249258, doi:10.1175/JAS-D-15-0382.1, 2017a.

Igel, A. L. and van den Heever, S. C.: The Importance of the Shape of Cloud Droplet Size Distributions in Shallow Cumulus Clouds. Part II: Bulk Microphysics Simulations,. J. Atmos. Sci. 74, 259 273, doi:10.1175/JAS-D-15-0383.1, 2017b.

Khain, A., Pokrovsky, A., Pinsky, M., Seifert, A., and Phillips, V.: Simulation of Effects of Atmospheric Aerosols on Deep Turbulent Convective Clouds Using a Spectral Microphysics Mixed-Phase Cumulus Cloud Model. Part I: Model Description and Possible Applications, J. Atmos. Sci., 61, 2963-2982, doi:10.1175/JAS-3350.1, 2004.

Khain, A. P., Beheng, K. D., Heymsfield, A., Korolev, A., Krichak, S. O., Levin, Z., Pinsky, M., Phillips, V., Prabhakaran, T., Teller, A., van den Heever, S. C., and Yano, J.-I.: Representation of microphysical processes in cloud-resolving models: Spectral (bin) microphysics versus bulk parameterization, Rev. Geophys., 53, 247-322, doi:10.1002/2014RG000468, 2015.
Kumjian, M. R., Ganson, S. M., and Ryzhkov, A. V.: Freezing of Raindrops in Deep Convective Updrafts: A Microphysical and Polarimetric Model, J. Atmos. Sci., 69, 3471-3490, doi:10.1175/JAS-D-12-067.1, 2012.

McFarquhar, G. M., Hsieh, T.-L., Freer, M., Mascio, J., and Jewett, B. F.: The Characterization of Ice Hydrometeor Gamma Size Distributions as Volumes in $\mathrm{N} 0-\lambda-\mu$ Phase Space: Implications for Microphysical Process Modeling, J. Atmos. Sci., 72, 892-909, doi:10.1175/JAS-D-14-0011.1, 2015.

Milbrandt, J. A. and McTaggart-Cowan, R.: Sedimentation-Induced Errors in Bulk Microphysics Schemes, J. Atmos. Sci., 67, 39313948, doi:10.1175/2010JAS3541.1, 2010.

Milbrandt, J. A. and Yau, M. K.: A Multimoment Bulk Microphysics Parameterization. Part I: Analysis of the Role of the Spectral Shape Parameter, J. Atmos. Sci., 62, 3051-3064, doi:10.1175/JAS3534.1, 2005.

Miles, N. L., Verlinde, J., and Clothiaux, E. E.: Cloud Droplet Size Distributions in Low-Level Stratiform Clouds, J. Atmos. Sci., 57, 295-311, doi:10.1175/1520-0469(2000)057< 0295:CDSDIL> 2.0.CO;2, 2000.

Morrison, H. and Grabowski, W. W.: Comparison of Bulk and Bin Warm-Rain Microphysics Models Using a Kinematic Framework, J. Atmos. Sci., 64, 2839-2861, doi:10.1175/JAS3980, 2007.

Rotstayn, L. D. and Liu, Y.: Sensitivity of the First Indirect Aerosol Effect to an Increase of Cloud Droplet Spectral Dispersion with Droplet Number Concentration, J. Clim., 16, 3476-3481, doi:10.1175/1520-0442(2003)016<3476:SOTFIA> 2.0.CO;2, 2003.

Saleeby, S. M. and Cotton, W. R.: A Large-Droplet Mode and Prognostic Number Concentration of Cloud Droplets in the Colorado State University Regional Atmospheric Modeling System (RAMS). Part I: Module Descriptions and Supercell Test Simulations, J. Appl. Meteorol., 43, 182-195, doi:10.1175/15200450(2004)043< 0182:ALMAPN> 2.0.CO;2, 2004.

Saleeby, S. M. and van den Heever, S. C.: Developments in the CSU-RAMS Aerosol Model: Emissions, Nucleation, Regeneration, Deposition, and Radiation, J. Appl. Meteorol. Clim., 52, 2601-2622, doi:10.1175/JAMC-D-12-0312.1, 2013.

Seifert, A. and Beheng, K. D.: A double-moment parameterization for simulating autoconversion, accretion and selfcollection, Atmos. Res., 59-60, 265-281, doi:10.1016/S0169-8095(01)001260,2001 .

Walko, R. L., Cotton, W. R., Feingold, G., and Stevens, B.: Efficient computation of vapor and heat diffusion between hydrometeors in a numerical model, Atmos. Res., 53, 171-183, doi:10.1016/S0169-8095(99)00044-7, 2000.

Zhu, P. and Albrecht, B.: Large eddy simulations of continental shallow cumulus convection, J. Geophys. Res., 108, 4453, doi:10.1029/2002JD003119, 2003. 